\title{
Diagonal invariants and the refined multimahonian distribution
}

\author{
Fabrizio Caselli
}

Received: 1 July 2008 / Accepted: 10 October 2008 / Published online: 3 November 2008

(C) Springer Science+Business Media, LLC 2008

\begin{abstract}
Combinatorial aspects of multivariate diagonal invariants of the symmetric group are studied. As a consequence we deduce the existence of a multivariate extension of the classical Robinson-Schensted correspondence. Further byproducts are a purely combinatorial algorithm to describe the irreducible decomposition of the tensor product of two irreducible representations of the symmetric group, and new symmetry results on permutation enumeration with respect to descent sets.
\end{abstract}

Keywords Diagonal invariants - Symmetric groups · Descent sets · Hilbert series · Kronecker coefficients.

\section{Introduction}

The invariant theory of finite groups generated by reflections has attracted many mathematicians since their classification in the works of Chevalley [10] and Shepard and Todd [28] with a particular attention on the combinatorial aspects of it. This is mainly due to the fact that the study of invariant and coinvariant algebras by means of generating functions leads naturally to nontrivial combinatorial properties of finite reflection groups. A crucial example in this context which is a link between the invariant theory and the combinatorics of the symmetric group is the Robinson-Schensted correspondence. This correspondence (see $[11,26]$ ) is a bijection between the symmetric group on $n$ elements and the set of ordered pairs of standard tableaux with $n$ boxes with the same shape. This is based on the row bumping algorithm and was originally introduced by Robinson to study the Littlewood-Richardson rule and by Schensted to study the lengths of increasing subsequences of a word. This algorithm

F. Caselli (凶)

Dipartimento di Matematica, Università di Bologna, Piazza di Porta San Donato 5, Bologna 40126, Italy

e-mail: caselli@dm.unibo.it 
has found applications in the representation theory of the symmetric group, in the theory of symmetric functions and the theory of the plactic monoid. Moreover, it is certainly fascinating from a combinatorial point of view and has inspired a considerable number of papers in the last decades. This correspondence has been generalized to other Weyl groups, by defining ad hoc tableaux, or to semistandard tableaux in the so-called RSK-correspondence, by considering permutations as special matrices with nonnegative integer entries.

The main goal of this work is to exploit further the relationship between the Robinson-Schensted correspondence and the theory of invariants of the symmetric group. By interpreting the Hilbert series with respect to a multipartition degree of certain (diagonal) invariant and coinvariant algebras in terms of (descents of) tableaux and permutations we deduce the existence of a multivariate extension of the Robinson-Schensted correspondence, which is based on the decomposition of tensor products of irreducible representations of the symmetric group. The idea of a relation between diagonal invariants and tensor product multiplicities for a finite subgroup of $G L(V)$ goes back to Solomon (see [29, Remark 5.14]) and pervades the results of Gessel [19] on multipartite $P$-partitions. Although we can not define this correspondence explicitly, we can deduce from it an explicit combinatorial algorithm to describe the irreducible decomposition of the tensor product of two irreducible representations of the symmetric group. Finally, we show some further consequences in the theory of permutation enumeration.

\section{Background}

Let $V$ be a finite dimensional $\mathbb{C}$-vector space and $W$ be a finite subgroup of the general linear group $G L(V)$ generated by reflections, i.e. elements of finite order that fix a hyperplane pointwise. We refer to such a group simply as a reflection group. The most significant example of such a group is the symmetric group acting by permuting a fixed linear basis of $V$. Other important examples are Weyl groups acting on the corresponding root space. In this paper we concentrate on the case of the symmetric groups (and some other related groups). Nevertheless, we preserve the symbol $W$ to denote the symmetric group $S_{n}$ on the $n$-element set $[n] \stackrel{\text { def }}{=}\{1,2, \ldots, n\}$.

Given a permutation $\sigma \in W$ we denote by

$$
\operatorname{Des}(\sigma) \stackrel{\text { def }}{=}\{i \in[n-1]: \sigma(i)>\sigma(i+1)\}
$$

the (right) descent set of $\sigma$ and its major index by

$$
\operatorname{maj}(\sigma) \stackrel{\text { def }}{=} \sum_{i \in \operatorname{Des}(\sigma)} i .
$$

For example if $\sigma=35241$ we have $\operatorname{Des}(\sigma)=\{2,4\}$ and maj $(\sigma)=6$. We recall the following equidistribution result due to MacMahon (see [22]). 
Theorem 2.1 We have

$$
\begin{aligned}
W(q) & \stackrel{\text { def }}{=} \sum_{\sigma \in W} q^{\operatorname{maj}(\sigma)}=\sum_{\sigma \in W} q^{\operatorname{inv}(\sigma)} \\
& =\prod_{i=1}^{n}\left(1+q+q^{2}+\cdots+q^{i}\right),
\end{aligned}
$$

where $\operatorname{inv}(\sigma)=\mid\{(i, j): i<j$ and $\sigma(i)>\sigma(j)\} \mid$ is the number of inversions of $\sigma$.

The dual action of a reflection group on $V^{*}$ can be extended to the symmetric algebra $S\left(V^{*}\right)$ of polynomial functions on $V$. If we fix a basis of $V$, the symmetric algebra is naturally identified with the algebra of polynomials $\mathbb{C}[X]$. Here and in what follows we use the symbol $X$ to denote an $n$-tuple of variables $X=\left(x_{1}, \ldots, x_{n}\right)$. The symmetric group $W$ acts on $\mathbb{C}[X]$ by permuting the variables. As customary, we denote by $\mathbb{C}[X]^{W}$ the ring of invariant polynomials (fixed points of the action of $W$ ). We also denote by $I_{+}^{W}$ the ideal of $\mathbb{C}[X]$ generated by homogeneous polynomials in $\mathbb{C}[X]^{W}$ of strictly positive degree. The coinvariant algebra associated to $W$ is defined as the corresponding quotient algebra

$$
R^{W} \stackrel{\text { def }}{=} \mathbb{C}[X] / I_{+}^{W} .
$$

The coinvariant algebra has important applications in the representation theory since it is isomorphic to the group algebra of $W$ (as $W$-modules) and in the topology of the flag variety since it is isomorphic to its cohomology ring.

If $R$ is a multigraded $\mathbb{C}$-vector space we can record the dimensions of its homogeneous components via its Hilbert series

$$
\operatorname{Hilb}(R)\left(q_{1}, \ldots, q_{k}\right) \stackrel{\text { def }}{=} \sum_{a_{1}, \ldots, a_{k} \in \mathbb{N}} \operatorname{dim}\left(R_{a_{1}, \ldots, a_{k}}\right) q_{1}^{a_{1}} \cdots q_{k}^{a_{k}},
$$

where $R_{a_{1}, \ldots, a_{k}}$ is the homogeneous subspace of $R$ of multidegree $\left(a_{1}, \ldots, a_{k}\right)$.

We note that, since the ideal $I_{+}^{W}$ is generated by homogeneous polynomials (by total degree) the coinvariant algebra is graded in $\mathbb{N}$. It turns out that the polynomial $W(q)$ appearing in Theorem 2.1 is the Hilbert series of the coinvariant algebra $R^{W}$ :

$$
W(q)=\operatorname{Hilb}\left(R^{W}\right)(q) .
$$

This is a crucial example of interplay between the invariant theory of $W$ and the combinatorics of $W$ (by Theorem 2.1). All the other cases considered in this paper are algebraic and combinatorial variations and generalizations of this fundamental fact.

The coinvariant algebra affords also the structure of a multigraded vector space which refines the structure of graded algebra. This further decomposition can be described in terms of descents of permutations and descents of tableaux and was originally obtained in a work of Adin, Brenti and Roichman [2] for Weyl groups of type $A$ and $B$ (see also [9] for Weyl groups of type $D$ and [4] for other complex reflection groups). 
If $M$ is a monomial in $\mathbb{C}[X]$ we denote by $\lambda(M)$ its exponent partition, i.e. the partition obtained by rearranging the exponents of $M$. We say that a polynomial is homogeneous of partition degree $\lambda$ if it is a linear combination of monomials whose exponent partition is $\lambda$. We note that the exponent partition is not well-defined in the coinvariant algebra. For example, for $n=3$ the monomials $x_{1}^{2}$ and $x_{2} x_{3}$ are in the same class in the coinvariant algebra (since $x_{1}^{2}-x_{2} x_{3}=x_{1}\left(x_{1}+x_{2}+x_{3}\right)-$ $\left.\left(x_{1} x_{2}+x_{1} x_{3}+x_{2} x_{3}\right)\right)$, though they have distinct exponent partitions. Nevertheless the exponent partition will be fundamental in defining a "partition degree" also in the coinvariant algebra.

We recall the definition of the dominance order in the set of partitions of $n$. We write $\mu \unlhd \lambda$, and we say that $\mu$ is smaller than or equal to $\lambda$ in the dominance order, if $\mu_{1}+\cdots+\mu_{i} \leq \lambda_{1}+\cdots+\lambda_{i}$ for all $i$. We write $\mu \triangleleft \lambda$ if $\mu \unlhd \lambda$ and $\mu \neq \lambda$. We let $R_{\lambda}^{(1)}$ be the subspace of $R^{W}$ consisting of elements that can be represented as a linear combination of monomials with exponent partition smaller than or equal to $\lambda$ in dominance order. We also denote by $R_{\lambda}^{(2)}$ the subspace of $R^{W}$ consisting of elements that can be represented as a linear combination of monomials with exponent partition strictly smaller than $\lambda$ in dominance order. The subspaces $R_{\lambda}^{(1)}$ and $R_{\lambda}^{(2)}$ are also $W$-submodules of $R^{W}$ and we denote their quotient by

$$
R_{\lambda} \stackrel{\text { def }}{=} R_{\lambda}^{(1)} / R_{\lambda}^{(2)} .
$$

The $W$-modules $R_{\lambda}$ provide a further decomposition of the homogeneous components of the coinvariant algebra $R^{W}$ (see [2, Theorem 3.12]).

Theorem 2.2 There exists an isomorphism of $W$-modules

$$
\varphi: R_{k}^{W} \cong \bigoplus_{|\lambda|=k} R_{\lambda},
$$

such that $\varphi^{-1}\left(R_{\lambda}\right)$ can be represented by homogeneous polynomials of partition degree $\lambda$.

We can use this result to define a partition degree on the coinvariant algebra: we simply say that an element in $R_{k}^{W}$ is homogeneous of partition degree $\lambda$ if its image under the isomorphism $\varphi$ is in $R_{\lambda}$. We can therefore define the Hilbert polynomial of $R^{W}$ with respect to the partition degree by

$$
\operatorname{Hilb}\left(R^{W}\right)\left(q_{1}, \ldots, q_{n}\right)=\sum_{\lambda}\left(\operatorname{dim} R_{\lambda}\right) q_{1}^{\lambda_{1}} \cdots q_{n}^{\lambda_{n}} .
$$

The dimensions of the $W$-modules $R_{\lambda}$ can be easily described in terms of descents of permutations. Given $\sigma \in W$ we define a partition $\lambda(\sigma)$ be letting

$$
\lambda(\sigma)_{i}=|\operatorname{Des}(\sigma) \cap\{i, \ldots, n\}| .
$$

Note that the knowledge of $\lambda(\sigma)$ is equivalent to the knowledge of $\operatorname{Des}(\sigma)$ and that $\operatorname{maj}(\sigma)=|\lambda(\sigma)|$. Then we have the following result which can be viewed as a refine- 
ment of Equation (1) (see [2, Corollary 3.11]):

$$
\operatorname{dim} R_{\lambda}=|\{\sigma \in W: \lambda(\sigma)=\lambda\}|
$$

Once we know the dimensions of the $W$-modules $R_{\lambda}$ we may wonder about their irreducible decomposition. For this reason we introduce the refined fake degree polynomial $f^{\mu}\left(q_{1}, \ldots, q_{n}\right)$ as the polynomial whose coefficient of $q_{1}^{\lambda_{1}} \cdots q_{n}^{\lambda_{n}}$ is the multiplicity of the representation $\mu$ in $R_{\lambda}$ if $\lambda$ is a partition, and zero otherwise, i.e.

$$
f^{\mu}(Q)=\sum_{\lambda}\left\langle\chi^{\mu}, \chi^{R_{\lambda}}\right\rangle Q^{\lambda}
$$

where $Q^{\lambda} \stackrel{\text { def }}{=} q_{1}^{\lambda_{1}} \cdots q_{n}^{\lambda_{n}}$. In this formula we denote by $\chi^{\rho}$ the character of a representation $\rho$ and by $\langle\cdot, \cdot\rangle$ the scalar product on the space of class functions on $W$ with respect to which the characters of the irreducible representations form an orthonormal basis. The polynomials $f^{\mu}(Q)$ have a very simple combinatorial interpretation based on standard tableaux that we are going to describe.

Given a partition $\mu$ of $n$, the Ferrers diagram of shape $\mu$ is a collection of boxes, arranged in left-justified rows, with $\mu_{i}$ boxes in row $i$. A standard tableau of shape $\mu$ is a filling of the Ferrers diagram of shape $\mu$ using the numbers from 1 to $n$, each occurring once, in such way that rows are increasing from left to right and columns are increasing from top to bottom. We denote by $\mathcal{S T}$ the set of standard tableaux with $n$ boxes. For example the following picture

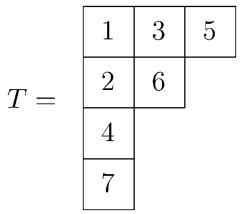

represents a standard tableau of shape $(3,2,1,1)$. We say that $i$ is a descent of a standard tableau $T$ if $i$ appears strictly above $i+1$ in $T$. We denote by $\operatorname{Des}(T)$ the set of descents of $T$ and we let $\operatorname{maj}(T)$ be the sum of its descents. Finally, we denote by $\mu(T)$ the shape of $T$. In the previous example we have $\operatorname{Des}(T)=\{1,3,5,6\}$ and so $\operatorname{maj}(T)=15$. As we did for permutations, given a tableau $T$ we define a partition $\lambda(T)$ by putting

$$
(\lambda(T))_{i}=|\operatorname{Des}(T) \cap\{i, \ldots, n\}| .
$$

It is known that irreducible representations of the symmetric group $W$ are indexed by partitions of $n$. We therefore use the same symbol $\mu$ to denote a partition or the corresponding Specht module. The following result appearing in [2, Theorem 4.1] describes explicitly the decomposition into irreducibles of the $W$-modules $R_{\lambda}$ and refines a well-known result on the irreducible decomposition of the homogeneous components of $R^{W}$ attributed to Lusztig (unpublished) and to Kraśkiewicz and Wey$\operatorname{man}([21])$. 
Theorem 2.3 The multiplicity of the representation $\mu$ in $R_{\lambda}$ is

$$
\mid\{T \text { tableau }: \mu(T)=\mu \text { and } \lambda(T)=\lambda\} \mid
$$

and so

$$
f^{\mu}\left(q_{1}, \ldots, q_{n}\right)=\sum_{\{T: \mu(T)=\mu\}} Q^{\lambda(T)} .
$$

\section{Refined multimahonian distributions}

We let $\mathbb{C}\left[X_{1}, \ldots, X_{k}\right]$ be the algebra of polynomials in the $n k$ variables $x_{i, j}$, with $i \in[k]$ and $j \in[n]$, i.e. we use the capital variable $X_{i}$ for the $n$-tuple of variables $x_{i, 1}, \ldots, x_{i, n}$. We consider the natural action of $W^{k}$ and of its diagonal subgroup $\Delta W$ on $\mathbb{C}\left[X_{1}, \ldots, X_{k}\right]$. By means of the above decomposition of the coinvariant algebra we can also decompose the algebra

$$
\frac{\mathbb{C}\left[X_{1}, \ldots, X_{k}\right]}{I_{+}^{W^{k}}} \cong \underbrace{R^{W} \otimes \cdots \otimes R^{W}}_{k}
$$

in homogeneous components whose degrees are $k$-tuples of partitions with at most $n$ parts. In particular we say that an element in $\mathbb{C}\left[X_{1}, \ldots, X_{k}\right] / I_{+}^{W^{k}}$ is homogeneous of multipartition degree $\left(\lambda^{(1)}, \ldots, \lambda^{(k)}\right)$ if it belongs to $R_{\lambda^{(1)}}^{W} \otimes \cdots \otimes R_{\lambda^{(k)}}^{W}$ by means of the above mentioned canonical isomorphism. We are mainly interested in the subalgebra

$$
\left(\frac{\mathbb{C}\left[X_{1}, \ldots, X_{k}\right]}{I_{+}^{W^{k}}}\right)^{\Delta W} \cong \frac{\mathbb{C}\left[X_{1}, \ldots, X_{k}\right]^{\Delta W}}{J_{+}^{W^{k}}} .
$$

Here $J_{+}^{W^{k}}$ denotes the ideal generated by totally invariant polynomials with no constant term inside $\mathbb{C}\left[X_{1}, \ldots, X_{k}\right]^{\Delta W}$ and the isomorphism is due to the fact that the operator

$$
F \mapsto F \stackrel{\text { def }}{=} \frac{1}{|W|} \sum_{\sigma \in \Delta W} \sigma(F)
$$

is an inverse of the natural projection $\mathbb{C}\left[X_{1}, \ldots, X_{k}\right]^{\Delta W} / J_{+}^{W^{k}} \rightarrow\left(\mathbb{C}\left[X_{1}, \ldots, X_{k}\right] /\right.$ $\left.I_{+}^{W^{k}}\right)^{\Delta W}$.

We can therefore consider the Hilbert polynomial

$$
\begin{aligned}
& \operatorname{Hilb}\left(\frac{\mathbb{C}\left[X_{1}, \ldots, X_{k}\right]^{\Delta W}}{J_{+}^{W^{k}}}\right) \\
& \stackrel{\text { def }}{=} \sum_{\lambda^{(1)}, \ldots, \lambda^{(k)}} \operatorname{dim}\left(\frac{\mathbb{C}\left[X_{1}, \ldots, X_{k}\right]^{\Delta W}}{J_{+}^{W^{k}}}\right)_{\lambda^{(1)}, \ldots, \lambda^{(k)}} Q_{1}^{\lambda^{(1)}} \cdots Q_{k}^{\lambda^{(k)}} .
\end{aligned}
$$


In this formula the symbol $Q_{i}$ stands for the $n$-tuple of variables $q_{i, 1}, \ldots, q_{i, n}$ and the sum is over all partitions $\lambda^{(1)}, \ldots, \lambda^{(k)}$ with at most $n$ parts.

Our next target is to describe the previous Hilbert series. For this we need to introduce one further ingredient. We define the Kronecker coefficients of $W$ by

$$
\begin{aligned}
& g_{\mu^{(1)}, \ldots, \mu^{(k)}} \stackrel{\text { def }}{=} \frac{1}{|W|} \sum_{\sigma \in W} \chi^{\mu^{(1)}}(\sigma) \cdots \chi^{\mu^{(k)}}(\sigma) \\
&=\left\langle\chi^{\mu^{(1)}} \cdots \chi^{\mu^{(k-1)}}, \chi^{\mu^{(k)}}\right\rangle_{W},
\end{aligned}
$$

(recalling that $\chi(\sigma)=\chi\left(\sigma^{-1}\right)$ ) where $\mu^{(1)}, \ldots, \mu^{(k)}$ are irreducible representations of $W$. In other words $g_{\mu^{(1)}}, \ldots, \mu^{(k)}$ is the multiplicity of $\mu^{(k)}$ in the (reducible) representation $\mu^{(1)} \otimes \cdots \otimes \mu^{(k-1)}$. These numbers have been deeply studied in the literature (see, e.g., [7, 13, 23, 25]) though they do not have an explicit description such as a combinatorial interpretation. A consequence of our main result is also a recursive combinatorial definition of the numbers $g_{\mu^{(1)}}, \ldots, \mu^{(k)}$ which is independent of the character theory of $W$.

Now we can state the following result which relates the Hilbert series above with Kronecker coefficients and the refined fake degree polynomials.

Theorem 3.1 We have

$$
\begin{aligned}
\operatorname{Hilb} & \left(\mathbb{C}\left[X_{1}, \ldots, X_{k}\right]^{\Delta W} / J_{+}^{W^{k}}\right)\left(Q_{1}, \ldots, Q_{k}\right) \\
= & \sum_{\mu^{(1)}, \ldots, \mu^{(k)}} g_{\mu^{(1)}, \ldots, \mu^{(k)}} f^{\mu^{(1)}}\left(Q_{1}\right) \cdots f^{\mu^{(k)}}\left(Q_{k}\right) \\
= & \sum_{T_{1}, \ldots, T_{k}} g_{\mu\left(T_{1}\right), \ldots, \mu\left(T_{k}\right)} Q_{1}^{\lambda\left(T_{1}\right)} \cdots Q_{k}^{\lambda\left(T_{k}\right)}
\end{aligned}
$$

Proof The first equality is essentially an application of a result of Solomon (see [29, Theorem 5.11]). If $G$ is a finite group and $V$ is a graded $G$-module, then Solomon's result expresses the Hilbert series of the diagonal invariants in the $k$-th tensor power of $V$ in terms of the irreducible decomposition of (the homogeneous components of) $V$. One can easily verify that this result holds also if $V$ is a multigraded $G$-module and so we can apply it to the coinvariant algebra of $W$ considered as a multigraded $W$-module and obtain the first equality.

The second equality follows directly from Theorem 2.3.

We recall that the algebra $\mathbb{C}\left[X_{1}, \ldots, X_{k}\right]^{\Delta W}$, being Cohen-Macaulay (see [30, Proposition 3.1]), is a free module over its subalgebra $\mathbb{C}\left[X_{1}, \ldots, X_{k}\right]^{W^{k}}$. This implies directly that if we consider $\mathbb{C}\left[X_{1}, \ldots, X_{k}\right]$ as an algebra graded in $\mathbb{N}^{k}$ in the natural way, then,

$$
\operatorname{Hilb}\left(\mathbb{C}\left[X_{1}, \ldots, X_{k}\right]^{\Delta W} / J_{+}^{W^{k}}\right)\left(q_{1}, \ldots, q_{k}\right)=\frac{\operatorname{Hilb}\left(\mathbb{C}\left[X_{1}, \ldots, X_{k}\right]^{\Delta W}\right)\left(q_{1}, \ldots, q_{k}\right)}{\operatorname{Hilb}\left(\mathbb{C}\left[X_{1}, \ldots, X_{k}\right]^{W^{k}}\right)\left(q_{1}, \ldots, q_{k}\right)}
$$


Now, the algebra $\mathbb{C}\left[X_{1}, \ldots, X_{k}\right]$ is also multigraded by $k$-tuples of partitions with at most $n$ parts: we just say that a monomial $M$ is homogeneous of multipartition degree $\left(\lambda^{(1)}, \ldots, \lambda^{(k)}\right)$ if its exponent partition with respect to the variables $x_{i, 1}, \ldots x_{i, n}$ is $\lambda^{(i)}$ for all $i$. We write in this case $\lambda^{(i)}(M) \stackrel{\text { def }}{=} \lambda^{(i)}$ for all $i$ and $\Lambda(M) \stackrel{\text { def }}{=}\left(\lambda^{(1)}, \ldots, \lambda^{(k)}\right)$. The refinement of Equation (2) using the Hilbert series with respect to multipartition degree is no longer implied by the Cohen-Macaulayness of $\mathbb{C}\left[X_{1}, \ldots, X_{k}\right]^{\Delta W}$. For this we need to use the existence of the representations $R_{\lambda}$ in a more subtle way. Given $\sigma \in W$ we define a monomial

$$
a_{\sigma}=\prod x_{\sigma(i)}^{\lambda(\sigma)_{i}}
$$

By definition we clearly have $\lambda\left(a_{\sigma}\right)=\lambda(\sigma)$. In [18] and [2] it is proved that the set of monomials $\left\{a_{\sigma}: \sigma \in W\right\}$ is a basis for the coinvariant algebra $R^{W}$. The proof in [2] is based on a straightening law. For its description we need to introduce an ordering on the set of monomials of the same degree: for $m$ and $m^{\prime}$ monomials of the same total degree in $\mathbb{C}[X]$ we let $m \prec m^{\prime}$ if

1. $\lambda(m) \triangleleft \lambda\left(m^{\prime}\right)$; or

2. $\lambda(m)=\lambda\left(m^{\prime}\right)$ and $\operatorname{inv}(\pi(m))>\operatorname{inv}\left(\pi\left(m^{\prime}\right)\right)$,

where $\pi(m)$ is the permutation $\pi$ having a minimal number of inversions such that the exponent in $m$ of $x_{\pi(i)}$ is greater than or equal to the exponent in $m$ of $x_{\pi(i+1)}$ for all $i$. The straightening law is the following: let $m$ be a monomial in $\mathbb{C}[X]$. Then $\mu:=\lambda(m)-\lambda(\pi(m))$ is still a partition and

$$
m=m_{\mu} \cdot a_{\pi(m)}+\sum_{m^{\prime} \prec m} c_{m, m^{\prime}} m^{\prime}
$$

where $c_{m, m^{\prime}} \in \mathbb{C}$ and $m_{\mu}$ is the monomial symmetric function. The straightening algorithm stated in [2] uses elementary symmetric functions instead of monomial symmetric functions, but one can easily check that the two statements are equivalent. The fact that the set $\left\{a_{\sigma}: \sigma \in W\right\}$ is a basis of $R^{W}$ implies directly that the set of monomials

$$
a_{\sigma_{1}, \ldots, \sigma_{k}} \stackrel{\text { def }}{=} a_{\sigma_{1}}\left(X_{1}\right) \cdots a_{\sigma_{k}}\left(X_{k}\right)
$$

is a basis for the coinvariant algebra of $W^{k}$, i.e. the algebra $\mathbb{C}\left[X_{1}, \ldots, X_{k}\right] / I_{+}^{W^{k}}$. Now, the monomials $a_{\sigma_{1}, \ldots, \sigma_{k}}$ form a basis for the algebra $\mathbb{C}\left[X_{1}, \ldots X_{k}\right]$ as a free module over the subring $\mathbb{C}\left[X_{1}, \ldots, X_{k}\right]^{W^{k}}$ of $W^{k}$-invariants (being a basis of the coinvariant algebra $\left.\mathbb{C}\left[X_{1}, \ldots, X_{k}\right] / I_{+}^{W^{k}}\right)$, i.e.

$$
\mathbb{C}\left[X_{1}, \ldots, X_{k}\right]=\bigoplus_{\sigma_{1}, \ldots, \sigma_{k} \in W} \mathbb{C}\left[X_{1}, \ldots, X_{k}\right]^{W^{k}} a_{\sigma_{1}, \ldots, \sigma_{k}} .
$$

The following result states a triangularity property of this basis. If $\left(\mu^{(1)}, \ldots, \mu^{(k)}\right)$ and $\left(\lambda^{(1)}, \ldots, \lambda^{(k)}\right)$ are two $k$-tuples of partitions we write $\left(\mu^{(1)}, \ldots, \mu^{(k)}\right) \unlhd$ $\left(\lambda^{(1)}, \ldots, \lambda^{(k)}\right)$ if $\mu^{(i)} \unlhd \lambda^{(i)}$ for all $i$ and we denote by $\mathbb{C}\left[X_{1}, \ldots, X_{k}\right]_{\unlhd\left(\lambda^{(1)}, \ldots, \lambda^{(k)}\right)}$ the 
space of polynomials spanned by monomials with multipartition degree $\unlhd\left(\lambda^{(1)}, \ldots\right.$, $\left.\lambda^{(k)}\right)$. We similarly define $\mathbb{C}\left[X_{1}, \ldots, X_{k}\right]_{\triangleleft\left(\lambda^{(1)}, \ldots, \lambda^{(k)}\right)}$.

Lemma 3.2 Let $M \in \mathbb{C}\left[X_{1}, \ldots, X_{k}\right]$ be a monomial and let

$$
M=\sum_{\sigma_{1}, \ldots, \sigma_{k} \in W} f_{\sigma_{1}, \ldots, \sigma_{k}} a_{\sigma_{1}, \ldots, \sigma_{k}},
$$

where $f_{\sigma_{1}, \ldots, \sigma_{k}} \in \mathbb{C}\left[X_{1}, \ldots, X_{k}\right]^{W^{k}}$. Then this sum is restricted to those $\sigma_{1}, \ldots, \sigma_{k}$ such that $\Lambda(M)-\Lambda\left(a_{\sigma_{1}, \ldots, \sigma_{k}}\right)$ is a $k$-tuple of partitions and

$$
f_{\sigma_{1}, \ldots, \sigma_{k}} \in \mathbb{C}\left[X_{1}, \ldots, X_{k}\right] \unlhd \Lambda(M)-\Lambda\left(a_{\sigma_{1}, \ldots, \sigma_{k}}\right) .
$$

Proof Given two monomials $M=m_{1}\left(X_{1}\right) \cdots m_{k}\left(X_{k}\right)$ and $M^{\prime}=m_{1}^{\prime}\left(X_{1}\right) \cdots m_{k}^{\prime}\left(X_{k}\right)$ we let $M \prec M^{\prime}$ if $m_{i} \prec m_{i}^{\prime}$ for all $i$. We proceed by a double induction on the total degree and on $\prec$ within the set of monomials of the same multidegree. If $M$ has total degree zero the result is trivial. Otherwise let $\Lambda(M)=\left(\lambda^{(1)}, \ldots, \lambda^{(k)}\right)$. If $M$ is minimal with respect to the ordering $\prec$ then $\lambda^{(i)}$ is minimal with respect to the dominance order for all $i$. If there exists $i$ such that $\left|\lambda^{(i)}\right| \geq n$ then $M=\left(x_{i, 1} \cdots x_{i, n}\right) M^{\prime}$ and the result follows by induction since the total degree of $M^{\prime}$ is strictly smaller than the degree of $M$. If $\left|\lambda^{(i)}\right|<n$ for all $i$ then $\lambda^{(i)}=\left(1^{k_{i}}\right)$ by the minimality condition. Then $m_{i}=a_{\pi\left(m_{i}\right)}$ for all $i$ and the result follows. In the general case we can apply the straightening law (3) to all the $m_{i}$ 's getting

$$
M=f_{M} \cdot a_{\pi\left(m_{1}\right), \ldots, \pi\left(m_{k}\right)}+\sum_{M^{\prime} \prec M} c_{M, M^{\prime}} M^{\prime},
$$

where $f_{M}$ is a homogeneous $W^{k}$-invariant polynomial of multipartition degree $\Lambda(M)-\Lambda\left(a_{\pi\left(m_{1}\right), \ldots, \pi\left(m_{k}\right)}\right)$. Then the result follows by induction.

Now recall the already mentioned sequence of isomorphisms of $W$-modules

$$
\begin{aligned}
\frac{\mathbb{C}\left[X_{1}, \ldots, X_{k}\right]^{\Delta W}}{J_{+}^{W^{k}}} & \cong\left(\frac{\mathbb{C}\left[X_{1}, \ldots, X_{k}\right]}{I_{+}^{W^{k}}}\right)^{\Delta W} \cong(\underbrace{R^{W} \otimes \cdots \otimes R^{W}}_{k \text { times }})^{\Delta W} \\
& \cong \bigoplus_{\lambda^{(1)}, \ldots, \lambda^{(k)}}\left(R_{\lambda^{(1)}} \otimes \cdots \otimes R_{\lambda^{(k)}}\right)^{\Delta W} .
\end{aligned}
$$

Consider a basis of $\left(R_{\lambda^{(1)}} \otimes \cdots \otimes R_{\lambda^{(k)}}\right)^{\Delta W}$. Every element of such a basis can be represented by a homogeneous element in $\mathbb{C}\left[X_{1}, \ldots, X_{k}\right]$ of multipartition degree $\left(\lambda^{(1)}, \ldots, \lambda^{(k)}\right)$ (by definition) which is invariant for the action of $\Delta W$. In fact, if a representative $F$ of a basis element is not $\Delta W$-invariant we can consider its symmetrization $F^{\#}$ since, clearly, $F$ and $F^{\#}$ represents the same class in $\left(R_{\lambda^{(1)}} \otimes \cdots \otimes R_{\lambda^{(k)}}\right)^{\Delta W}$. We denote by $\mathcal{B}\left(\lambda^{(1)}, \ldots, \lambda^{(k)}\right)$ this set of representatives, i.e. $\mathcal{B}\left(\lambda^{(1)}, \ldots, \lambda^{(k)}\right)$ is a set of polynomials in $\mathbb{C}\left[X_{1}, \ldots, X_{k}\right]^{\Delta W}$ of multipartition degree $\left(\lambda^{(1)}, \ldots, \lambda^{(k)}\right)$ whose corresponding classes form a basis of $\left(R_{\lambda^{(1)}} \otimes \cdots \otimes\right.$ 
$\left.R_{\lambda^{(k)}}\right)^{\Delta W}$. We denote by $\mathcal{B}$ the (disjoint) union of all $\mathcal{B}\left(\lambda^{(1)}, \ldots, \lambda^{(k)}\right)$. By the CohenMacaulayness of $\mathbb{C}\left[X_{1}, \ldots, X_{k}\right]^{\Delta W}$ we can deduce that the set $\mathcal{B}$ is a basis for $\mathbb{C}\left[X_{1}, \ldots, X_{k}\right]^{\Delta W}$ as a free $\mathbb{C}\left[X_{1}, \ldots, X_{k}\right]^{W^{k}}$-module (see [30, Proposition 3.1]), i.e.

$$
\mathbb{C}\left[X_{1}, \ldots, X_{k}\right]^{\Delta W}=\bigoplus_{b \in \mathcal{B}} \mathbb{C}\left[X_{1}, \ldots, X_{k}\right]^{W^{k}} \cdot b .
$$

The following result implies a crucial triangularity property of the basis $\mathcal{B}$.

Lemma 3.3 Let $F \in \mathbb{C}\left[X_{1}, \ldots, X_{k}\right]^{\Delta W}$ be homogeneous of multipartition degree $\Lambda(F)$. Then the unique expression

$$
F=\sum_{b \in \mathcal{B}} f_{b} b
$$

with $f_{b} \in \mathbb{C}\left[X_{1}, \ldots, X_{k}\right]^{W^{k}}$ for all $b \in \mathcal{B}$, is such that the sum is restricted to those $b \in \mathcal{B}$ for which $\Lambda(F)-\Lambda(b)$ is a $k$-tuple of partitions and

$$
f_{b} \in \mathbb{C}\left[X_{1}, \ldots, X_{k}\right]_{\unlhd \Lambda(F)-\Lambda(b)}^{W^{k}} .
$$

Proof Let $\prec$ be a total order on the set of $k$-tuples of partitions of length at most $n$ satysfying the following two conditions

- If $\left|\mu^{(1)}\right|+\cdots+\left|\mu^{(k)}\right|<\left|\lambda^{(1)}\right|+\cdots+\left|\lambda^{(k)}\right|$ then $\left(\mu^{(1)}, \ldots, \mu^{(k)}\right) \prec\left(\lambda^{(1)}, \ldots, \lambda^{(k)}\right)$;

- If $\mu^{(i)} \unlhd \lambda^{(i)}$ for all $i$, then $\left(\mu^{(1)}, \ldots, \mu^{(k)}\right) \prec\left(\lambda^{(1)}, \ldots, \lambda^{(k)}\right)$.

We proceed by induction on the multipartition degree of $F$ with respect to the total order $\prec$. If $F$ has degree zero then the result is trivial. Otherwise let $\Lambda(F)=$ $\left(\lambda^{(1)}, \ldots, \lambda^{(k)}\right)$ be the multipartition degree of $F$. Then $F$ represents an element in $\left(R_{\lambda(1)} \otimes \cdots \otimes R_{\lambda(k)}\right)^{\Delta W}$. Therefore,

$$
F=\sum_{b \in \mathcal{B}\left(\lambda^{(1)}, \ldots, \lambda^{(k)}\right)} c_{b} b
$$

in $\left(R_{\lambda^{(1)}} \otimes \cdots \otimes R_{\lambda^{(k)}}\right)^{\Delta W}$. This means that

$$
F=\sum_{b \in \mathcal{B}\left(\lambda^{(1)}, \ldots, \lambda^{(k)}\right)} c_{b} b+G
$$

in $(\underbrace{R^{W} \otimes \cdots \otimes R^{W}}_{k \text { times }})^{\Delta W}$, where $G$ is a $\Delta W$-invariant polynomial such that

$$
G \in \mathbb{C}\left[X_{1}, \ldots, X_{k}\right]_{\triangleleft \Lambda(F)}^{\Delta W} .
$$

Finally we deduce from this that

$$
F=\sum_{b \in \mathcal{B}\left(\mu^{(1)}, \ldots, \mu^{(k)}\right)} c_{b} b+G+H
$$


in $\mathbb{C}\left[X_{1}, \ldots, X_{k}\right]^{\Delta W}$, where $H$ belongs to $I_{+}^{W^{k}}$. We can clearly assume that $G$ and $H$ are homogeneous with the same total multidegree of $F$. The induction hypothesis applies directly to $G$. Regarding $H$, by Lemma 3.2, we can express $H=$ $\sum_{\sigma_{1}, \ldots, \sigma_{k}} f_{\sigma_{1}, \ldots, \sigma_{k}} a_{\sigma_{1}, \ldots, \sigma_{k}}$ with $f_{\sigma_{1}, \ldots, \sigma_{k}} \in \mathbb{C}\left[X_{1}, \ldots, X_{k}\right]_{\unlhd \Lambda(F)-\Lambda\left(a_{\sigma_{1}, \ldots, \sigma_{k}}\right)}^{W^{k}}$ since $H$ is a sum of monomials of multipartition degree smaller than or equal to $\Lambda(F)$ in dominance order. Moreover, all the polynomials $f_{\sigma_{1}, \ldots, \sigma_{k}}$ have positive degree since $H \in I_{+}^{W^{k}}$. Now we can apply the operator \# to this identity and we get

$$
H=\sum_{\sigma_{1}, \ldots, \sigma_{k}} f_{\sigma_{1}, \ldots, \sigma_{k}} a_{\sigma_{1}, \ldots, \sigma_{k}}^{\#} .
$$

Finally we can apply our induction hypothesis to the polynomials $a_{\sigma_{1}, \ldots, \sigma_{k}}^{\#}$ since they have degree smaller than $F$ and the proof is completed by observing that, clearly,

$$
\mathbb{C}\left[X_{1}, \ldots, X_{k}\right]_{\unlhd \Lambda} \cdot \mathbb{C}\left[X_{1}, \ldots, X_{k}\right]_{\unlhd \Lambda^{\prime}} \subseteq \mathbb{C}\left[X_{1}, \ldots, X_{k}\right]_{\unlhd \Lambda+\Lambda^{\prime}}
$$

We observe that Lemma 3.3 fails to be true for a generic homogeneous basis $\mathcal{B}$ of $\mathbb{C}\left[X_{1}, \ldots, X_{k}\right]^{\Delta W}$ as a free $\mathbb{C}\left[X_{1}, \ldots, X_{k}\right]^{W^{k}}$-module. We refer the reader to [24, Section 4.2], [3] and [6] for the explicit description of some bases of $\mathbb{C}\left[X_{1}, \ldots, X_{k}\right]^{\Delta W}$ over $\mathbb{C}\left[X_{1}, \ldots, X_{k}\right]^{W^{k}}$, with particular attention to the case $k=2$.

For notational convenience, if $\Lambda=\left(\lambda^{(1)}, \ldots, \lambda^{(k)}\right)$ is a multipartition, we denote by

$$
\mathcal{Q}^{\Lambda} \stackrel{\text { def }}{=} Q_{1}^{\lambda^{(1)}} \cdots Q_{k}^{\lambda^{(k)}}=\prod_{i=1}^{k} \prod_{j=1}^{n} q_{i, j}^{\lambda_{j}^{(i)}} .
$$

Corollary 3.4 We have

$$
\begin{aligned}
\operatorname{Hilb}\left(\frac{\mathbb{C}\left[X_{1}, \ldots, X_{k}\right]^{\Delta W}}{J_{+}^{W^{k}}}\right)\left(Q_{1}, \ldots, Q_{k}\right) & =\frac{\operatorname{Hilb}\left(\mathbb{C}\left[X_{1}, \ldots, X_{k}\right]^{\Delta W}\right)\left(Q_{1}, \ldots, Q_{k}\right)}{\operatorname{Hilb}\left(\mathbb{C}\left[X_{1}, \ldots, X_{k}\right]^{W^{k}}\right)\left(Q_{1}, \ldots, Q_{k}\right)} \\
& =\sum_{b \in \mathcal{B}} \mathcal{Q}^{\Lambda(b)}
\end{aligned}
$$

Proof The fact that $\operatorname{Hilb}\left(\mathbb{C}\left[X_{1}, \ldots, X_{k}\right]^{\Delta W} / J_{+}^{W^{k}}\right)\left(Q_{1}, \ldots, Q_{k}\right)=\sum_{b \in \mathcal{B}} \mathcal{Q}^{\Lambda(b)}$ is clear from the definition of the multipartition degree on $\mathbb{C}\left[X_{1}, \ldots, X_{k}\right]^{\Delta W} / J_{+}^{W^{k}}$ and the definition of the set $\mathcal{B}$. Lemma 3.3 implies that

$$
\operatorname{dim} \mathbb{C}\left[X_{1}, \ldots, X_{k}\right]_{\unlhd \Lambda}^{\Delta W}=\sum_{b \in \mathcal{B}\left\{\Lambda^{\prime}: \Lambda^{\prime}+\Lambda(b) \unlhd \Lambda\right\}} \operatorname{dim} \mathbb{C}\left[X_{1}, \ldots, X_{k}\right]_{\Lambda^{\prime}}^{W^{k}},
$$


and similarly with $\triangleleft$ instead of $\unlhd$. Therefore

$$
\begin{aligned}
\operatorname{dim} \mathbb{C}\left[X_{1}, \ldots, X_{k}\right]_{\Lambda}^{\Delta W} & =\operatorname{dim} \mathbb{C}\left[X_{1}, \ldots, X_{k}\right]_{\unlhd \Lambda}^{\Delta W}-\operatorname{dim} \mathbb{C}\left[X_{1}, \ldots, X_{k}\right]_{\triangleleft \Lambda}^{\Delta W} \\
& =\sum_{b \in \mathcal{B}\left\{\Lambda^{\prime}: \Lambda^{\prime}+\Lambda(b)=\Lambda\right\}} \sum_{\operatorname{dim} \mathbb{C}\left[X_{1}, \ldots, X_{k}\right]_{\Lambda^{\prime}}^{W^{k}}} .
\end{aligned}
$$

Note that in the last sum there is only one summand corresponding to $\Lambda^{\prime}=\Lambda-\Lambda(b)$ if this is a multipartition, and there are no summands otherwise. So we have

$$
\begin{aligned}
\operatorname{Hilb}\left(\mathbb{C}\left[X_{1}, \ldots, X_{k}\right]^{\Delta W}\right) & =\sum_{\Lambda} \operatorname{dim} \mathbb{C}\left[X_{1}, \ldots, X_{k}\right]_{\Lambda}^{\Delta W} \mathcal{Q}^{\Lambda} \\
& =\sum_{\Lambda} \sum_{b \in \mathcal{B}\left\{\Lambda^{\prime}: \Lambda^{\prime}+\Lambda(b)=\Lambda\right\}} \operatorname{dim} \mathbb{C}\left[X_{1}, \ldots, X_{k}\right]_{\Lambda^{\prime}}^{W^{k}} \mathcal{Q}^{\Lambda} \\
& =\sum_{b \in \mathcal{B}} \sum_{\Lambda^{\prime}} \operatorname{dim} \mathbb{C}\left[X_{1}, \ldots, X_{k}\right]_{\Lambda^{\prime}}^{W^{k}} \mathcal{Q}^{\Lambda^{\prime}+\Lambda(b)} \\
& =\sum_{b \in \mathcal{B}} \mathcal{Q}^{\Lambda(b)} \sum_{\Lambda^{\prime}} \operatorname{dim} \mathbb{C}\left[X_{1}, \ldots, X_{k}\right]_{\Lambda^{\prime}}^{W^{k}} \mathcal{Q}^{\Lambda^{\prime}} \\
& =\sum_{b \in \mathcal{B}} \mathcal{Q}^{\Lambda(b)} \operatorname{Hilb}\left(\mathbb{C}\left[X_{1}, \ldots, X_{k}\right]^{W^{k}}\right) .
\end{aligned}
$$

Now we need to study the two Hilbert series of the invariant algebras $\mathbb{C}\left[X_{1}, \ldots, X_{k}\right]^{\Delta W}$ and $\mathbb{C}\left[X_{1}, \ldots, X_{k}\right]^{W^{k}}$ with respect to the multipartition degree. Before stating our next result we need to recall a classical theorem that can be attributed to Gordon [20] and Garsia and Gessel [16] on multipartite partitions. We say that a collection $\left(f^{(1)}, \ldots, f^{(k)}\right)$ of $k$ elements of $\mathbb{N}^{n}$ is a $k$-partite partition if $f_{j}^{(i)} \geq f_{j+1}^{(i)}$ whenever $f_{j}^{(h)}=f_{j+1}^{(h)}$ for all $h<i$. For notational convenience we denote by $\left.W^{(k)} \stackrel{\text { def }}{=}\left\{\left(\sigma_{1}, \ldots, \sigma_{k}\right) \in W^{k}: \sigma_{1} \cdots \sigma_{k}=1\right)\right\}$. The main property of $k$-partite partitions that we need is the following.

Theorem 3.5 There exists a bijection between the set of $k$-partite partitions and the set of $2 k$-tuples $\left(\sigma_{1}, \ldots, \sigma_{k}, \mu^{(1)}, \ldots, \mu^{(k)}\right)$ such that

- $\left(\sigma_{1}, \ldots, \sigma_{k}\right) \in W^{(k)}$;

- $\mu^{(i)}$ is a partition with at most $n$ parts;

- $\mu_{j}^{(i)}>\mu_{j+1}^{(i)}$ whenever $j \in \operatorname{Des}\left(\sigma_{i}\right)$.

The bijection is such that $\mu^{(i)}$ is obtained by reordering the coefficients of $f^{(i)}$.

We can now prove the following formula for the quotient of the Hilbert polynomials with respect to the multipartition degree associated to the invariant algebras of $\Delta W$ and $W^{k}$. 
Theorem 3.6 We have

$$
\frac{\operatorname{Hilb}\left(\mathbb{C}\left[X_{1}, \ldots, X_{k}\right]^{\Delta W}\right)\left(Q_{1}, \ldots, Q_{k}\right)}{\operatorname{Hilb}\left(\mathbb{C}\left[X_{1}, \ldots, X_{k}\right]^{W^{k}}\right)\left(Q_{1}, \ldots, Q_{k}\right)}=\sum_{\left(\sigma_{1}, \ldots, \sigma_{k}\right) \in W^{(k)}} Q_{1}^{\lambda\left(\sigma_{1}\right)} \cdots Q_{k}^{\lambda\left(\sigma_{k}\right)}
$$

Proof We observe that the set of monomials $X_{1}^{f^{(1)}} \cdots X_{k}^{f^{(k)}}$ as $\left(f^{(1)}, \ldots, f^{(k)}\right)$ varies among all possible $k$-partite partitions is a set of representatives for the orbits of the action of $\Delta W$ in the set of monomials in $\mathbb{C}\left[X_{1}, \ldots, X_{k}\right]$. By means of Theorem 3.5 we can deduce that

$$
\operatorname{Hilb}\left(\mathbb{C}\left[X_{1}, \ldots, X_{k}\right]^{\Delta W}\right)\left(Q_{1}, \ldots, Q_{k}\right)=\sum_{\substack{\sigma_{1}, \ldots, \sigma_{k}, \mu^{(1)}, \ldots, \mu^{(k)}}} Q_{1}^{\mu^{(1)}} \cdots Q_{k}^{\mu^{(k)}},
$$

where the indices in the previous sum are such that they satisfy the conditions stated in Theorem 3.5. We now observe that we have an equivalence of conditions

$$
\mu_{j}^{(i)}>\mu_{j+1}^{(i)} \text { whenever } j \in \operatorname{Des}\left(\sigma_{i}\right) \Longleftrightarrow \mu^{(i)}-\lambda\left(\sigma_{i}\right) \text { is a partition. }
$$

Therefore we can simplify the previous sum in the following way

$$
\sum_{\substack{\sigma_{1}, \ldots, \sigma_{k}, \mu^{(1)}, \ldots, \mu^{(k)}}} Q_{1}^{\mu^{(1)}} \cdots Q_{k}^{\mu^{(k)}}=\sum_{\left(\sigma_{1}, \ldots, \sigma_{k}\right) \in W^{(k)}} \sum_{v^{(1)}, \ldots, \nu^{(k)}} Q_{1}^{v^{(1)}+\lambda\left(\sigma_{1}\right)} \cdots Q_{k}^{\nu^{(k)}+\lambda\left(\sigma_{k}\right)}
$$

where the last sum is on all possible $k$-tuples of partitions $v^{(1)}, \ldots, v^{(k)}$ of length at most $n$. The result follows since, clearly,

$$
\operatorname{Hilb}\left(\mathbb{C}\left[X_{1}, \ldots, X_{k}\right]^{W^{k}}=\sum_{v^{(1)}, \ldots, v^{(k)}} Q_{1}^{v^{(1)}} \cdots Q_{k}^{v^{(k)}}\right.
$$

Putting all these results together we obtain the following sequence of equivalent interpretations for what we may call the refined multimahonian distribution.

Theorem 3.7 We have

$$
\begin{aligned}
W\left(Q_{1}, \ldots, Q_{k}\right) & \stackrel{\text { def }}{=} \sum_{T_{1}, \ldots, T_{k}} g_{\mu\left(T_{1}\right), \ldots, \mu\left(T_{k}\right)} Q_{1}^{\lambda\left(T_{1}\right)}, \ldots, Q_{k}^{\lambda\left(T_{k}\right)} \\
& =\sum_{\mu^{(1)}, \ldots, \mu^{(k)}} g_{\mu^{(1)}, \ldots, \mu^{(k)}} f^{\mu^{(1)}}\left(Q_{1}\right) \cdots f^{\mu^{(k)}}\left(Q_{k}\right) \\
& \left.=\operatorname{Hilb}\left(\mathbb{C}\left[X_{1}, \ldots, X_{k}\right]^{\Delta W} / J_{+}^{W}\right)\right)\left(Q_{1}, \ldots, Q_{k}\right)
\end{aligned}
$$




$$
\begin{aligned}
& =\frac{\operatorname{Hilb}\left(\mathbb{C}\left[X_{1}, \ldots, X_{k}\right]^{\Delta W}\right)\left(Q_{1}, \ldots, Q_{k}\right)}{\operatorname{Hilb}\left(\mathbb{C}\left[X_{1}, \ldots, X_{k}\right]^{W^{k}}\right)\left(Q_{1}, \ldots, Q_{k}\right)} \\
& =\sum_{\sigma_{1} \cdots \sigma_{k}=1} Q_{1}^{\lambda\left(\sigma_{1}\right)} \cdots Q_{k}^{\lambda\left(\sigma_{k}\right)}
\end{aligned}
$$

Proof The four identities are the contents of Theorem 3.1, Corollary 3.4 and Theorem 3.6.

The cardinality of the set of $k$-tuples of permutations in $W^{(k)}$ having fixed descent sets was already studied by Gessel in [19] and the idea to use Kronecker products is already present in his work. As pointed out by Reiner one can obtain an alternative proof of the equality between the first and the last line in Theorem 3.7 starting from Gessel's result [19, Theorem 17]. The crucial point in this alternative proof is the observation that the symmetric functions appearing in Gessel's theorem are the images under the characteristic map of the characters of the ribbon representations (whose characters can be expressed in terms of standard tableaux by means of the Young's rule). He also remarked that all the equalities appearing in Theorem 3.7 can also be proved and reformulated in terms of the Stanley-Reisner ring $\mathbb{C}\left[\Delta_{n}\right]$ of the simplicial complex $\Delta_{n}$, which is the barycentric subdivision of an $(n-1)$-dimensional simplex (see, e.g., [24, Corollary 4.2.4] for the equivalent reformulation of the equality between the last two lines in Theorem 3.7 in this context). This is essentially due to the fact that $\mathbb{C}\left[\Delta_{n}\right]$ is isomorphic to $\mathbb{C}[X]$ as a multigraded $W$-module. Nevertheless, this isomorphism is no longer true for Weyl groups of other types and the Stanley-Reisner ring is not defined at all for general complex reflection groups. This is the reason why we think that the approach through diagonal invariants in polynomial algebras can be better in view of a possible generalization of these results to other groups.

The reason why we call the distribution $W\left(Q_{1}, \ldots, Q_{k}\right)$ refined is that one can consider its coarse version $W\left(q_{1}, \ldots q_{k}\right)$ obtained by putting $q_{i, j}=q_{i}$ for all $i$ and $j$. In this case one obtains the so-called multimahonian distribution $\sum_{\left(\sigma_{1}, \ldots, \sigma_{k}\right) \in W^{(k)}} q_{1}^{\operatorname{maj}\left(\sigma_{1}\right)} \cdots q_{k}^{\operatorname{maj}\left(\sigma_{k}\right)}$ which has been extensively studied in the literature (see, e.g., $[1,5,8,15,16])$.

Corollary 3.8 There exists a map $\mathcal{T}: W^{(k)} \longrightarrow \mathcal{S} \mathcal{T}^{k}$ satisfying the following two conditions:

1. For every $k$-tuple of tableaux $\left(T_{1}, \ldots, T_{k}\right)$,

$$
\left|\mathcal{T}^{-1}\left(T_{1}, \ldots, T_{k}\right)\right|=g_{\mu\left(T_{1}\right), \ldots, \mu\left(T_{k}\right)} .
$$

In particular it depends only on the shapes of the tableaux $T_{1}, \ldots, T_{k}$;

2. if $\mathcal{T}\left(\sigma_{1}, \ldots, \sigma_{k}\right)=\left(T_{1}, \ldots, T_{k}\right)$ then $\operatorname{Des}\left(T_{i}\right)=\operatorname{Des}\left(\sigma_{i}\right)$ for all $i=1, \ldots, k$.

The classical Robinson-Schensted correspondence (see $[32, \S 7.11]$ for a description of this correspondence) provides a bijective proof of this corollary in the case $k=2$. 
We can also conjecture the existence of a correspondence $\mathcal{T}$ that satisfies the conditions of Corollary 3.8 and is well-behaved with respect to cyclic permutations of the arguments in the sense that

$$
\mathcal{T}\left(\sigma_{1}, \ldots, \sigma_{k}\right)=\left(T_{1}, \ldots, T_{k}\right) \Longrightarrow \mathcal{T}\left(\sigma_{2}, \ldots, \sigma_{k}, \sigma_{1}\right)=\left(T_{2}, \ldots, T_{k}, T_{1}\right) .
$$

One can also ask whether the correspondence $\mathcal{T}$ can be chosen to have the following symmetry property

$$
\mathcal{T}\left(\sigma_{1}, \ldots, \sigma_{k}\right)=\left(T_{1}, \ldots, T_{k}\right) \Longrightarrow \mathcal{T}\left(w_{0} \sigma_{1} w_{0}, \ldots, w_{0} \sigma_{k} w_{0}\right)=\left(T_{1}^{J}, \ldots, T_{k}^{J}\right),
$$

where $T \mapsto T^{J}$ denotes Schützenberger evacuation of the standard tableau $T$ (see $[15,27]$ and Proposition 4.6).

Problem 3.9 Find a map $\mathcal{T}$ as in Corollary 3.8 explicitly.

We observe that the resolution of this Problem would provide also an explicit combinatorial interpretation for the coefficients $g_{\mu^{(1)}, \ldots, \mu^{(k)}}$.

\section{Combinatorial applications}

In this final section we deduce some combinatorial results on Kronecker coefficients and permutation enumeration that follow from the results of the previous section. Our next goal is to show that we do not need to know the coefficients $g_{\mu^{(1)}, \ldots, \mu^{(k)}}$ to solve Problem 3.9. This is because Corollary 3.8 uniquely determines the coefficients $g_{\mu^{(1)}, \ldots, \mu^{(k)}}$ in the following sense.

Proposition 4.1 Let $\mathcal{T}: W^{(k)} \rightarrow \mathcal{S} \mathcal{T}^{k}$ be such that

1. $\left|\mathcal{T}^{-1}\left(T_{1}, \ldots, T_{k}\right)\right|$ depends uniquely on the shapes of $T_{1}, \ldots, T_{k}$;

2. if $\mathcal{T}\left(\sigma_{1}, \ldots, \sigma_{k}\right)=\left(T_{1}, \ldots, T_{k}\right)$ then $\operatorname{Des}\left(T_{i}\right)=\operatorname{Des}\left(\sigma_{i}\right)$ for all $i=1, \ldots, k$.

Then $\mathcal{T}$ satisfies the conditions of Corollary 3.8, i.e. $\left|\mathcal{T}^{-1}\left(T_{1}, \ldots, T_{k}\right)\right|=$ $g_{\mu\left(T_{1}\right), \ldots, \mu\left(T_{k}\right)}$ for all $\left(T_{1}, \ldots, T_{k}\right) \in \mathcal{S T}^{k}$.

This proposition is an immediate consequence of the following elementary results that we state without proof.

Lemma 4.2 Let $\mu=\left(\mu_{1}, \ldots, \mu_{r}\right)$ be a partition of $n$ (with $\left.\mu_{r}>0\right)$. Then

1. there exists a unique standard tableau $T_{\mu}$ of shape $\mu$ and descent set $\operatorname{Des}\left(T_{\mu}\right)=$ $\left\{\mu_{1}, \mu_{1}+\mu_{2}, \ldots, \mu_{1}+\cdots+\mu_{r-1}\right\}$

2. if $T$ is a standard tableau and $\operatorname{Des}(T)=\operatorname{Des}\left(T_{\mu}\right)$, then $\mu(T) \unrhd \mu$.

The unique tableau $T_{\mu}$ satisfying the conditions of Lemma 4.2 is called the row superstandard tableau of shape $\mu$ by Garsia and Maclarnan in [17] and is obtained by inserting the numbers form 1 to $\mu_{1}$ in the first row of the Ferrers diagram of $\mu$, then 
the numbers from $\mu_{1}+1$ to $\mu_{1}+\mu_{2}$ in the second row and so on in the following rows.

The following corollary is a recursion satisfied by the coefficients $g_{\mu^{(1)}, \ldots, \mu^{(k)}}$, it proves Proposition 4.1 and provides also an explicit entirely combinatorial algorithm

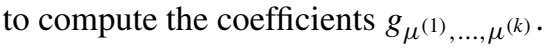

Corollary 4.3 Let $\mu^{(1)}, \ldots, \mu^{(k)}$ be a multipartition and let $D_{i}=\operatorname{Des}\left(T_{\mu^{(i)}}\right)$. Then

$$
\begin{aligned}
g_{\mu^{(1)}, \ldots, \mu^{(k)}} & \\
\left|\left\{\left(\sigma_{1}, \ldots, \sigma_{k}\right) \in W^{(k)}: \operatorname{Des}\left(\sigma_{i}\right)=D_{i}\right\}\right|- & \sum_{\left\{\begin{array}{c}
T_{1}, \ldots, T_{k} \in \mathcal{S} \mathcal{T}: \operatorname{Des}\left(T_{i}\right)=D_{i} \text { and } \\
\left(\mu\left(T_{1}\right), \ldots, \mu\left(T_{k}\right)\right) \triangleright\left(\mu^{(1)}, \ldots, \mu^{(k)}\right)
\end{array}\right\}} g_{\mu\left(T_{1}\right), \ldots, \mu\left(T_{k}\right)} .
\end{aligned}
$$

Proof By Corollary 3.8 we have

$$
\sum_{\substack{T_{1}, \ldots, T_{k} \in \mathcal{S} \mathcal{T}: \\ \operatorname{Des}\left(T_{i}\right)=D_{i}}} g_{\mu\left(T_{1}\right), \ldots, \mu\left(T_{k}\right)}=\left|\left\{\left(\sigma_{1}, \ldots, \sigma_{k}\right) \in W^{(k)}: \operatorname{Des}\left(\sigma_{i}\right)=D_{i}\right\}\right| .
$$

Now the claim follows by Lemma 4.2.

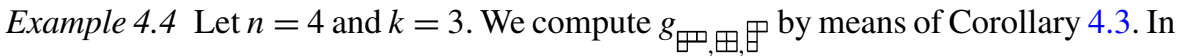
this case we have $D_{1}=\{3\}, D_{2}=\{2\}, D_{3}=\{2,3\}$. We have to determine all tableaux having these descents sets. Now we observe that the unique tableau having descent set $D_{1}$, is $T_{\square}$ and the unique tableau having descent set $D_{3}$ is $T_{\boxminus}$. On the other hand there are two tableaux having descent set $D_{2}$ and these are $T_{\boxplus}$ and \begin{tabular}{ll|l|l}
\hline$\frac{1}{3}$ & 2 & 4
\end{tabular} . So we have

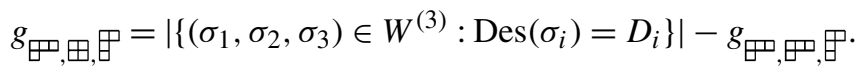

We observe that the cardinality of $\left\{\left(\sigma_{1}, \sigma_{2}, \sigma_{3}\right) \in W^{(3)}: \operatorname{Des}\left(\sigma_{i}\right)=D_{i}\right\}$ is 2 since the only two triplets in this set are $(1243,1423,1432)$ and $(2341,2413,2431)$. So we

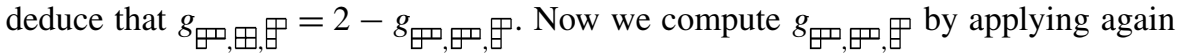
Corollary 4.3. In this case we have $D_{1}=D_{2}=\{3\}$ and $D_{3}=\{2,3\}$. By the previous observations on the tableaux having these descent sets we deduce that

$$
g_{\text {巴,巴, 田 }}=\left|\left\{\left(\sigma_{1}, \sigma_{2}, \sigma_{3}\right) \in W^{(3)}: \operatorname{Des}\left(\sigma_{i}\right)=D_{i}\right\}\right| .
$$

We can easily verify that the only element in this set is $(1342,1243,1432)$ and we can conclude that $g_{\boxplus, \boxplus, \boxplus}=1$.

It is clear that one can produce an algorithm to compute Kronecker coefficients based on Corollary 4.3, so some observations on the complexity bound for such algorithm are in order, at least in the case $k=3$. 
- Determination of the index set in the sum. Given an input set $D \subseteq\{n-1\}$, we need to compute, for any partition $\lambda$, the number of standard tableaux with descent set $D$ and shape $\lambda$. A naive way to do this is to list all standard tableaux having a given descent set $D$ and $n$ boxes. This can be done recursively by observing that deletion of the box containing $n$ in a tableau with descent set $D$ produces a tableau with $n-1$ boxes and descent set $D \backslash\{n-1\}$. This observation leads to a recursive algorithm of complexity $\Theta(|D| t(D))$ where $t(D)$ is the number of tableaux with at most $n$ boxes and descents prescribed by $D$. This can be bounded by $O\left(n^{2} T_{n}\right)$ where $T_{n}$ is the number of tableaux with $n$ boxes or equivalently the number of involutions in $W$.

- Determination of the number of recursion steps. A coarse bound for this is given by the number of triplets $\left(\lambda^{(1)}, \lambda^{(2)}, \lambda^{(3)}\right)$ with $\lambda^{(i)} \unrhd \mu^{(i)}$ and this is $O\left(p(n)^{3}\right)$ where $p(n)$ is the number of partitions of $n$.

- Determination of the cardinality of the set $\left\{\left(\sigma_{1}, \sigma_{2}, \sigma_{3}\right): \operatorname{Des}\left(\sigma_{i}\right)=D_{i}\right.$ and $\left.\sigma_{1} \sigma_{2} \sigma_{3}=1\right\}$. A naive approach to this problem would determine all permutations $\sigma_{1}$ having descent set $D_{1}$ and all permutations $\sigma_{2}$ having descent set $D_{2}$, and then verify for how many such pairs $\left(\sigma_{1}, \sigma_{2}\right)$ one has $\operatorname{Des}\left(\left(\sigma_{1} \sigma_{2}\right)^{-1}\right)=D_{3}$. Listing all permutations with a fixed descent set $D$ can be done with an algorithm of complexity $\Theta\left(n \beta_{n}(D)\right.$ ) where $\beta_{n}(D)=|\{\sigma \in W: \operatorname{Des}(\sigma)=D\}|$ (see [31, Example 2.2.4] for an explicit determinantal expression for the numbers $\left.\beta_{n}(D)\right)$ by means of the following observation. Given $D \subseteq[n-1]$, let $S=\left(s_{1}, \ldots, s_{n-1}\right)$ be the characteristic binary sequence of $D$, i.e. $s_{i}=1$ if $i \in D$ and $s_{i}=0$ otherwise. If we let $F(S, i) \stackrel{\text { def }}{=}\{\sigma \in W: \operatorname{Des}(\sigma)=D$ and $\sigma(i)=n\}$, then "deletion" of $n$ on elements in $F(S, i)$ produces all elements in

$$
\bigcup_{j}\left(F\left(\left(s_{1}, \ldots, \widehat{s_{i}}, \ldots, s_{n-1}\right), j\right) \cup F\left(\left(s_{1}, \ldots, \widehat{s_{i-1}}, \ldots, s_{n-1}\right), j\right)\right),
$$

where $\left.F\left(s_{1}, \ldots, \widehat{s_{i}}, \ldots, s_{n-1}\right), j\right) \stackrel{\text { def }}{=} \emptyset$ if $i=0, n$. The enumeration of how many pairs $\left(\sigma_{1}, \sigma_{2}\right)$ with $\operatorname{Des}\left(\sigma_{i}\right)=D_{i}$ satisfy $\operatorname{Des}\left(\left(\sigma_{1} \sigma_{2}\right)^{-1}\right)=D_{3}$ is an algorithm of complexity $\Theta\left(\beta_{n}\left(D_{1}\right) \beta_{n}\left(D_{2}\right) n\right)$. Now a simple observation that the average of the numbers $\beta_{n}(D)$ is $n ! / 2^{n-1}$ implies that such an algorithm to compute the Kronecker coefficients would be less efficient than the one shown by Derksen in [14] based on the Murnaghan-Nakayama rule. To improve the efficiency of an algorithm based on Corollary 4.3 one should be able to enumerate the elements in $\left\{\left(\sigma_{1}, \sigma_{2}, \sigma_{3}\right): \operatorname{Des}\left(\sigma_{i}\right)=D_{i}\right.$ and $\left.\sigma_{1} \sigma_{2} \sigma_{3}=1\right\}$ without listing them explicitly. A possible approach to this can be the following. Let

$$
\beta_{n}\left(D_{1}, D_{2}, D_{3}\right) \stackrel{\text { def }}{=} \mid\left\{\left(\sigma_{1}, \sigma_{2}, \sigma_{3}\right): \operatorname{Des}\left(\sigma_{i}\right)=D_{i} \text { and } \sigma_{1} \sigma_{2} \sigma_{3}=1\right\} \mid
$$

and

$$
\alpha_{n}\left(D_{1}, D_{2}, D_{3}\right) \stackrel{\text { def }}{=} \mid\left\{\left(\sigma_{1}, \sigma_{2}, \sigma_{3}\right): \operatorname{Des}\left(\sigma_{i}\right) \subseteq D_{i} \text { and } \sigma_{1} \sigma_{2} \sigma_{3}=1\right\} \mid
$$

Then the numbers $\beta_{n}$ can be expressed as an alternate sum of the numbers $\alpha_{n}$ by inclusion-exclusion and, as a direct consequence of Theorem 3.5, one can show 
that $\alpha_{n}\left(D_{1}, D_{2}, D_{3}\right)$ is equal to the number of 3-partite partitions whose $i$-th row is a reordering of the entries of the partition $\lambda\left(\sigma_{i}\right)$, where $\sigma_{i}$ is any element with descent set $D_{i}$. Then, if we let $\lambda\left(\sigma_{s}\right)=\left(r^{m_{s, r}}, \ldots, 2^{m_{s, 2}}, 1^{m_{s, 1}}\right)$, for $s=1,2,3$, one can easily deduce that $\alpha_{n}\left(D_{1}, D_{2}, D_{3}\right)$ is equal to the number of 3 dimensional arrays $\left(c_{i, j, k}\right)_{i, j, k \in[r]}$ with non negative integral coefficients satysfying the following conditions (see also Theorem 4.8 below):

$$
\sum_{i, j} c_{i, j, k}=m_{3, k}, \sum_{i, k} c_{i, j, k}=m_{2, j}, \sum_{j, k} c_{i, j, k}=m_{1, i}
$$

Next we show some new results on permutation statistics that can be deduced from Corollary 3.8. The first observation is a direct consequence of the symmetry of Kronecker coefficients with respect to their arguments. Let $D_{1}, \ldots, D_{k} \subseteq[n-1]$ and let $\pi$ be any permutation on $\{1, \ldots, k\}$. Then

$$
\left|\left\{\left(\sigma_{1}, \ldots, \sigma_{k}\right) \in W^{(k)}: \operatorname{Des}\left(\sigma_{i}\right)=D_{i}\right\}\right|=\left|\left\{\left(\sigma_{1}, \ldots, \sigma_{k}\right) \in W^{(k)}: \operatorname{Des}\left(\sigma_{i}\right)=D_{\pi(i)}\right\}\right| .
$$

This naturally leads to consider the following.

Problem 4.5 Find a combinatorial bijective proof for the identity (4).

It is plausible that the resolution of this problem could be a first step towards the resolution of Problem 3.9.

The classical Robinson-Schensted correspondence allows one to prove some results on permutation enumeration and in particular on the bimahonian distributions (see for example $[5,12,15])$. So, it is natural to ask how we can generalize these properties using the existence of the multivariate Robinson-Schensted correspondence. If $X$ is either a permutation on $n$ elements or a tableau with $n$ entries we denote by

$$
\begin{gathered}
\operatorname{Codes}(X) \stackrel{\text { def }}{=}\{i: n-i \in \operatorname{Des}(X)\} ; \\
\operatorname{Asc}(X) \stackrel{\text { def }}{=}[n-1] \backslash \operatorname{Des}(X) ; \\
\operatorname{Coasc}(X) \stackrel{\text { def }}{=}\{i: n-i \in \operatorname{Asc}(X)\} .
\end{gathered}
$$

The following is a multivariate generalization of a result of Foata and Schützenberger ([15, Theorem 2])

Proposition 4.6 For all $I \subset\{1, \ldots, k\}$ there exists an involution $F_{I}: W^{(k)} \rightarrow W^{(k)}$ such that, if $F_{I}\left(\sigma_{1}, \ldots, \sigma_{k}\right)=\left(\tau_{1}, \ldots, \tau_{k}\right)$, then

$$
\operatorname{Des}\left(\sigma_{i}\right)= \begin{cases}\operatorname{Codes}\left(\tau_{i}\right) & \text { if } i \in I \\ \operatorname{Des}\left(\tau_{i}\right) & \text { otherwise }\end{cases}
$$

Proof We recall (see $[15,27]$ ) that there exists an involution $T \mapsto T^{J}$ on the set of standard tableaux of the same shape such that $\operatorname{Des}(T)=\operatorname{Codes}\left(T^{J}\right)$. Then the result follows immediately from Corollary 3.9. 
It is clear that we can substitute in Proposition 4.6 Des with Asc and Codes with Coasc obtaining an analogous result. We can unify and generalize Equation (4) and Proposition 4.6 in the following statement.

Theorem 4.7 Fix $k$ subsets $D_{1}, \ldots, D_{k} \subseteq[n-1]$ arbitrarily. Then for any integer sequence $0 \leq i_{1} \leq i_{2} \leq i_{3} \leq k$ and for any permutation $\pi$ on $[k]$ the cardinality $C\left(i_{1}, i_{2}, i_{3} ; \pi\right)$ of the set

$$
\left\{\left(\sigma_{1}, \ldots, \sigma_{k}\right) \in W^{(k)}: D_{i}=\left\{\begin{array}{ll}
\operatorname{Des}\left(\sigma_{\pi(i)}\right) & \text { if } 0<i \leq i_{1}, \\
\operatorname{Codes}\left(\sigma_{\pi(i)}\right) & \text { if } i_{1}<i \leq i_{2}, \\
\operatorname{Asc}\left(\sigma_{\pi(i)}\right) & \text { if } i_{2}<i \leq i_{3}, \\
\operatorname{Coasc}\left(\sigma_{\pi(i)}\right) & \text { if } i_{3}<i \leq k,
\end{array}\right\}\right.
$$

depends only on the parity of $i_{2}$, and in particular it does not depend on the indices $i_{1}, i_{3}$ and on the permutation $\pi$.

Proof Since the knowledge of one of the three sets $\operatorname{Codes}(\sigma), \operatorname{Asc}(\sigma), \operatorname{Coasc}(\sigma)$ is equivalent to the knowledge of the set $\operatorname{Des}(\sigma)$ it is clear that, by (4), the number $C\left(i_{1}, i_{2}, i_{3} ; \pi\right)$ does not depend on $\pi$. So we can assume that $\pi=I d$. Then, from Proposition 4.6, we deduce that $C\left(i_{1}, i_{2}, i_{3} ; I d\right)$ depends only on $i_{2}$.

To prove that $C\left(i_{1}, i_{2}, i_{3} ; \pi\right)$ depends only on the parity of $i_{2}$ we consider the following permutation on $W^{(k)}$

$$
\left(\sigma_{1}, \ldots, \sigma_{k}\right) \mapsto\left(\sigma_{1} w_{0}, w_{0} \sigma_{2}, \ldots, \sigma_{2 h-1} w_{0}, w_{0} \sigma_{2 h}, \sigma_{2 h+1}, \ldots, \sigma_{k}\right),
$$

where $h$ is an integer such that $2 h \leq k$ and $w_{0}=(n, n-1, \ldots, 1)$ is the top element of $W$. Observing that $\operatorname{Des}(\sigma)=\operatorname{Coasc}\left(\sigma w_{0}\right)=\operatorname{Asc}\left(w_{0} \sigma\right)$ for all $\sigma \in W$, this map proves bijectively that $C(k, k, k, I d)=C(k-2 h, k-2 h, k-h, \pi)$, where $\pi=$ $(2 h+1,2 h+2, \ldots, k, 2,4, \ldots, 2 h, 1,3, \ldots, 2 h-1)$ and $C(k-1, k-1, k-1, I d)=$ $\left.C(k-1-2 h, k-1-2 h, k-1-h), \pi^{\prime}\right)$, where $\pi^{\prime}=(2 h+1,2 h+2, \ldots, k-$ $1,2,4, \ldots, 2 h, 1,3, \ldots, 2 h-1, k))$ and the proof is complete.

We conclude this paper with the following result that we state without proof. The Robinson-Schensted correspondence can be naturally generalized to the socalled RSK-correspondence (where the "K" stands for Knuth) between multisets with support in $\mathbb{N}^{2}$ of cardinality $n$ and pairs of semistandard tableaux of the same shape. One can show that an analogous correspondence exists also in the multivariate case between multisets with support in $\mathbb{N}^{k}$ of cardinality $n$ and $k$-tuples of semistandard Young tableaux of size $n$ preserving "descents". Here the descent sets $\operatorname{Des}_{1}(A), \ldots \operatorname{Des}_{k}(A)$ of a multiset $A$ are by definition the descent sets of the permutations appearing in Theorem 3.5 for the $k$-partite partition obtained reordering the elements of $A$. The descents of a semistandard tableau are the descents of its standardized tableau.

Theorem 4.8 There exists a correspondence $\mathcal{T}$ between multisets with support in $\mathbb{N}^{k}$ of cardinality $n$ and $k$-tuples of semistandard Young tableaux of size $n$ such that,

1. $\left|\mathcal{T}^{-1}\left(T_{1}, \ldots, T_{k}\right)\right|=g_{\mu\left(T_{1}\right), \ldots, \mu\left(T_{k}\right)}$; 
2. if $\mathcal{T}(A)=\left(T_{1}, \ldots, T_{k}\right)$ then the multiplicity of $i$ in $T_{j}$ is equal to the multiplicity of $i$ within the $j$-th coordinates of the elements of $A$ and

3. $\operatorname{Des}\left(T_{i}\right)=\operatorname{Des}_{i}(A)$.

Acknowledgement A particular gratitude goes to Vic Reiner for his precious comments on a preliminary draft of this paper.

\section{References}

1. Adin, R., Roichman, Y.: The flag major index and group actions on polynomial rings. Europ. J. Combin. 22, 431-446 (2001)

2. Adin, R., Brenti, F., Roichman, Y.: Descent representations and multivariate statistics. Trans. Amer. Math. Soc. 357(8), 3051-3082 (2005)

3. Allen, E.E.: The descent monomials and a basis for the diagonally symmetric polynomials. J. Algebraic Combin. 3, 5-16 (1994)

4. Bagno, E., Biagioli, R.: Colored-descent representations of complex reflection groups $G(r, p, n)$. Israel J. Math. 160, 317-347 (2007)

5. Barcelo, H., Reiner, V., Stanton, D.: Bimahonian distributions. J. London Math. Soc. 77, 627-646 (2008)

6. Bergeron, F., Lamontagne, F.: Decomposition of the diagonal action of $S_{n}$ on the coinvariant space of $S_{n} \times S_{n}$. Sém. Lothar. Combin. 52, B2e2 (2004/2007)

7. Bessenrodt, C., Kleshchev, A.: On Kronecker products of complex representations of the symmetric and alternating groups. Pacific J. Math. 190(2), 201-223 (1999)

8. Biagioli, R., Caselli, F.: Invariant algebras and major indices for classical Weyl groups. Proc. London Math. Soc. (3) 88(3), 603-631 (2004)

9. Biagioli, R., Caselli, F.: A descent basis for the coinvariant algebra of type D. J. Algebra 275, 517-539 (2004)

10. Chevalley, C.: Invariants of finite groups generated by reflections. Amer. J. Math. 77, 778-782 (1955)

11. de G. Robinson, B.: On the representations of the symmetric group. Amer. J. Math. 60(3), 745-760 (1938)

12. Désarménien, J., Foata, D.: Fonctions symétriques et séries hypergéométriques basiques multivariées. Bull. Soc. Math. France 113(1), 3-22 (1985)

13. Dvir, Y.: On the Kronecker product of $S_{n}$ characters. J. Algebra 154(1), 125-140 (1993)

14. Derksen, H.: Computing with characters of the symmetric groups, preprint available at www.math.lsa.umich.edu/ hderksen/preprints/sym.ps

15. Foata, D., Schützenberger, M.P.: Major index and inversion number of permutations. Math. Nachr. 83, 143-159 (1978)

16. Garsia, A., Gessel, I.: Permutation statistics and partitions. Adv. Math. 31, 288-305 (1979)

17. Garsia, A., McLarnan, T.: Relations between Young's natural and the Kazhdan-Lusztig representations of $S_{n}$. Adv. Math. 69, 32-92 (1988)

18. Garsia, A., Stanton, D.: Group actions of Stanley-Reisner rings and invariants of permutations groups. Adv. Math. 51, 107-201 (1984)

19. Gessel, I.: Multipartite P-partitions and inner products of skew Schur functions, Combinatorics and algebra. Contemp. Math., vol. 34, pp. 289-301. Amer. Math. Soc., Providence (1984)

20. Gordon, B.: Two theorems on multipartite partitions. J. London Math. Soc. 38, 459-464 (1963)

21. Kraśkiewicz, W., Weyman, J.: Algebra of coinvariants and the action of a Coxeter element. Bayreuth. Math. Schr. No. 63, 265-284 (2001)

22. MacMahon, P.A.: Combinatory analysis, vol. 1. Cambridge University Press, London (1915)

23. Regev, A.: On the height of the Kronecker product of $S_{n}$ characters. Israel J. Math. 42(1-2), 60-64 (1982)

24. Reiner, V.: Quotients of Coxeter complexes and $P$-partitions. Mem. Amer. Math. Soc. 95(460) (1992)

25. Rosas, M.H.: The Kronecker product of Schur functions indexed by two-row shapes or hook shapes. J. Algebraic Combin. 14, 153-173 (2001)

26. Schensted, C.: Longest increasing and decreasing subsequences. Canad. J. Math. 13, 179-191 (1961)

27. Schützenberger, M.P.: Quelques remarques sur une construction de Schensted. Math. Scand. 12, $117-$ 128 (1963) 
28. Shephard, G.C., Todd, J.A.: Finite unitary reflection groups. Canadian J. Math. 6, 274-304 (1954)

29. Solomon, L.: Partition identities and invariants of finite groups. J. Combin. Theory Ser. A 23, 148-175 (1977)

30. Stanley, R.P.: Invariants of finite groups and their applications to combinatorics. Bull. Amer. Math. Soc. 1, 475-511 (1979)

31. Stanley, R.P.: Enumerative combinatorics, vol. 1. Cambridge Studies in Advanced Mathematics, vol. 49. Cambridge University Press, Cambridge (1997)

32. Stanley, R.P.: Enumerative combinatorics, vol. 2. Cambridge Studies in Advanced Mathematics, vol. 62. Cambridge University Press, Cambridge (1999) 